\title{
Bellini's duct carcinoma: A report of two cases and a review of the literature
}

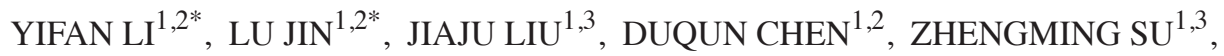 \\ LIQUN ZHOU ${ }^{4}$, BENTAO SHI ${ }^{1}$ and YONGQING LAI ${ }^{1}$ \\ ${ }^{1}$ Department of Urology, Peking University Shenzhen Hospital, Shenzhen, Guangdong 518036; \\ ${ }^{2}$ Department of Urology, Anhui Medical University, Hefei, Anhui 230032; \\ ${ }^{3}$ Department of Urology, Shantou University Medical College, Shantou, Guangdong 515041; \\ ${ }^{4}$ Department of Urology, Peking University First Hospital, Institute of Urology, Peking University, Beijing 100034, P.R. China
}

Received December 31, 2014; Accepted October 14, 2015

DOI: $10.3892 / \mathrm{ol} .2016 .4454$

\begin{abstract}
Bellini's duct carcinoma (BDC) is a rare and aggressive variant of renal cell carcinoma that possesses an extremely poor prognosis. The greater the grade or stage of disease, the poorer the prognosis tends to be. This study presents two cases of BDC; one case of low grade BDC and one case of high grade BDC in a 47-year-old male and 74-year-old female, respectively. The 47-year-old male patient presented with painless gross hematuria, which had lasted for 3 weeks and subsequently underwent purely laparoscopic nephroureterectomy. After 4-years of follow-up, the patient remained disease-free. By contrast, a right renal tumor was identified in the 74-year-old female patient during a routine examination. Radical right nephrectomy and lymph node dissection were performed, however, 10 months after surgery the patient succumbed due to wide-spread metastasis. The two cases reported in the present study not only represent excellent examples of the disease spectrum, but also act as a reminder of the possibility of detecting BDC in an early stage of disease. Therefore, the epidemiology of BDC has been discussed, and the aggressive growth pattern of BDC has been presented in terms of signs, symptoms and imaging examinations, including ultrasonography, computed tomography (CT), angiography and single photon emission CT, in the early stage of disease.
\end{abstract}

Correspondence to: Professor Yongqing Lai or Professor Bentao Shi, Department of Urology, Peking University Shenzhen Hospital, 1120 Lianhua Road, Shenzhen, Guangdong 518036, P.R. China

E-mail: yqlord@163.com

E-mail: shibentaopku@yahoo.com.cn

${ }^{*}$ Contributed equally

Key words: Bellini's duct carcinoma, epidemiology, early detection

\section{Introduction}

Bellini's duct carcinoma (BDC), which arises from the distal segment of the collecting duct, is a rare and aggressive malignant tumor with a poor prognosis. BDC comprises $<1 \%$ of all renal tumors (1), but it is extremely aggressive and is accompanied by metastatic diseases in the majority of reported cases. The prognosis of BDC is poor, with $\sim 70 \%$ patients succumbing due to disease progression within 2 years of diagnosis (2). The only favorable prognosis factor is low-grade and low-stage disease (3-5). If BDC could be detected in the early stage of disease, the poor prognosis of BDC may be changed. However, due to the rarity of BDC, the similarities of the symptoms, signs and imaging examination findings with other variants of renal cell carcinoma (RCC), and the poor prognosis of BDC, it is challenging and crucial to differentiate other RCC variants from BDC. The RCC database of Peking University Shenzhen Hospital (Shenzen, China) was reviewed, and out of a total of 311 RCC cases, two cases of BDC with different grades of disease and prognoses were identified.

\section{Case report}

Case one. In December 2010, a 47-year-old man presented to the Department of Urology, Peking University Shenzhen Hospital, with a complaint of painless gross hematuria during urination that had lasted 3 weeks. Physical examination revealed percussion pain over the left kidney region. The laboratory examinations revealed no extrarenal manifestations. The blood routine examination revealed no anemia and polycythemia; electrolyte examination revealed no hypercalcemia; erythrocyte sedimentation rate (ESR) examination revealed no fast ESR; and alkaline phosphatase examination revealed no elevated alkaline phosphatase. A kidney tumor was suspected based on the ultrasonography of the urinary system, which revealed a hypoechoic solid lesion in the left pelvis, measuring $3.76 \times 3.06 \mathrm{~cm}$ in size, which was heterogeneous inside and demonstrated a blood flow signal. Therefore, a contrast-enhanced computerized axial tomography (CT) scan was performed, which revealed a slightly contrasted, $2 \mathrm{~cm}$ in diameter, ill-defined mass with a complete capsule, 
in the middle and lower pole of the of the kidney. The CT scan confirmed that there was no tumor thrombus and no metastasis to the lymph nodes, liver, pancreas, spleen and other organs. The patient underwent purely laparoscopic nephroureterectomy.

The final pathological diagnosis was left renal BDC, with grade IV Fuhrman nuclear malignancy. According to the 2009 American Joint Committee on Cancer (AJCC) tumor-node-metastasis (TNM) classification of malignant tumors (6), the patient was diagnosed with stage T1aN0M0 disease, indicating low-grade BDC. Cytokeratin-7 (CK7) was not expressed in the tumor, but immunostaining for vimentin (Vim), epithelial membrane antigen (EMA), pan cytokeratin (AE1/AE3) and common acute lymphocytic leukemia antigen (CD10) was positive in the tumor. The patient rejected any type of chemotherapy when he was informed of the possible side effects. At the 4-year follow-up, the patient remained disease-free.

Case two. In addition, in September 2012, a 74-year-old woman presented to the Department of Urology, Peking University Shenzhen Hospital, with a hypoechoic solid lesion, $7 \mathrm{~cm}$ in diameter, on the right kidney, which was indicated during sonography. Urinary sonography indicated that the mass, which protruded outside the right kidney, was irregular in shape and heterogeneous inside, with an abundant blood flow signal. However, the physical examination was not notable and the laboratory examinations, which included routine blood and urine tests, blood electrolyte tests and ESR tests, as well as calcium and alkaline phosphatase tests, were normal. In order to further define the right renal mass, a contrast-enhanced CT was performed. The result confirmed a circular low-density shadow on the right kidney, which demonstrated contrast-enhancement in the early phase and was considered to mostly consist of renal cancer cells. According to the imaging findings and the patient's request, radical right nephrectomy and lymph node dissection was performed with a tentative diagnosis of renal cancer.

The tumor was morphologically consistent with BDC, measuring $7.8 \times 5.5 \times 5 \mathrm{~cm}$ in size, infiltrating perinephric fat and formatting satellite nodules in the kidney. One metastatic tumor was indicated in the right renal hilar lymph node. The final pathological diagnosis was right renal BDC, with grade IV Fuhrman nuclear malignancy. According to the 2009 AJCC TNM classification, the patient was diagnosed with stage T3aN1M0 disease, indicating high-grade BDC. Immunohistochemically, the tumor exhibited weak positive staining for Vim and CD10 and strong positive staining for AE1/AE3, CK7 and EMA. The findings were compatible with a diagnosis of BDC. The patient succumbed 10 months post-surgery due to widespread metastasis.

\section{Discussion}

The results demonstrated that the occurrence of BDC is $0.64 \%$, which is similar to other cases of BDC reported in Mexico, Japan and Italy $(5,7,8)$. The present two cases, which presented with different grades and prognoses of BDC, were in stark contrast. The TNM classifications of the low- and high-grade cases were T1aNOM0 and T3aN1M0, respectively. The high-grade case demonstrated a slight high stage with an infiltration of perinephric fat and only one regional lymph node metastasis; however, the patient with low-grade BDC survived for $\sim 5$ times the post-surgery lifespan of the patient with high-grade BDC. To the best of our knowledge, a lower grade and stage of disease is the only favorable prognosis factor for BDC.

Although $\mathrm{BDC}$ is a rare variant of RCC, BDC demonstrates the poorest prognosis and grows in a highly aggressive pattern, with early distant metastasis (9). Therefore, if BDC is detected at an early stage and intervention is prompt, the prognosis of BDC may be improved.

BDC is most common in older men ( $>40$ years old), with a mean age of diagnosis of 58.2 years; however, the majority of BDC patients are younger than classical RCC patients, which occurs predominantly in the seventh and eighth decades of life $(7,10,11)$. Auguet et al reported a case of BDC and highlighted the possibility of a strong family history of associated malignancy in BDC patients; however, this theory is controversial, as there is little supporting published data $(10,12)$. In the two cases reported in the present study, there was no family history of BDC. In one study, the majority of patients with BDC were symptomatic, with regional lymph node metastasis or distal metastasis at presentation (7). The majority of the complaints reported by patients with BDC, including flank pain, hematuria, palpable abdominal mass and weight loss, are similar to those reported by patients with conventional RCC. However, patients with BDC may also present with back pain (12), paraplegia (13), hemodialysis for chronic renal failure (14) and other symptoms associated with distal metastasis. BDC not only demonstrates an aggressive nature in the signs and symptoms of the disease, but also in imaging findings.

Clinically, B-scan ultrasonography is the first method selected to examine patients with renal cancer, and even for the census of renal tumors, due to its non-invasiveness, convenience and speed. In the two cases reported in the present study, ultrasonography of the patient with high-grade BDC revealed a more invasive tumor compared with the patient with low-grade BDC, with the mass being irregular in shape, protruding outside the right kidney and having an abundant blood flow signal. CT provides fundamental evidence for the diagnosis of renal cancer. Medullary involvement and an infiltrative appearance are common findings on CT scans and may indicate the diagnosis of collecting duct carcinoma. In large tumors, however, these features are frequently overshadowed by an exophytic or expansile component that may not be distinguished from the more common cortical renal cell carcinoma (15). In reported cases that used angiography, the BDCs were all hypovascular $(15,16)$, whereas $90 \%$ of clear cell RCCs were hypervascular (17). Wang et al first reported a BDC patient with deteriorated renal function, revealed by single photon emission CT, which is unusual in RCC (16). Wang et al (16) hypothesized that the infiltrative growth pattern of BDC impaired the nephrons. This is a hypothesis that requires additional studies.

In conclusion, $\mathrm{BDC}$ is a rare and aggressive variant of RCC. The two present low- and high-grade cases with favorable and poor prognoses, respectively, are examples of the disease spectrum and act as a reminder that certain invasive imaging evidence, including infiltrative appearance, medullary involvement and hypervascularity, may result in a poor prognosis. 


\section{Acknowledgements}

This work was supported by the National Natural Science Foundation of China (grant no. 81101922), the Science and Technology Development Fund Project of Shenzhen (grant nos. JCYJ20130402114702124 and JCYJ20150403091443329) and the fund of Guangdong Key medical subject.

\section{References}

1. Cohen HT and McGovern FJ: Renal-cell carcinoma. N Engl J Med 353: 2477-2490, 2005.

2. Miyake H, Haraguchi T, Takenaka A and Fujisawa M: Metastatic collecting duct carcinoma of the kidney responded to sunitinib. Int J Clin Oncol 16: 153-155, 2011.

3. Otani M, Shimizu T, Serizawa H, Ebihara Y and Nagashima Y: Low-grade renal cell carcinoma arising from the lower nephron: A case report with immunohistochemical, histochemical and ultrastructural studies. Pathol Int 51: 954-960, 2001.

4. Farah R, Ben-Izhak O, Munichor M and Cohen H: Low-grade renal collecting duct carcinoma. A case report with histochemical, immunohistochemical, and ultrastructural study. Ann Diagn Pathol 9: 46-48, 2005.

5. Vázquez-Lavista LG, Uribe-Uribe $\mathrm{N}$ and Gabilondo-Navarro F: Collecting duct renal cell carcinoma: Two different clinical stages, two different clinical outcomes. Urol Int 81: 116-118, 2008.

6. Edge SB, Byrd DR, Compton CC, Fritz AG, Greene FL and Trotti A (eds): Kidney. In: AJCC Cancer Staging Manual. 7th edition. Springer Verlag, New York, NY, pp479-489, 2010.

7. Tokuda N, Naito S, Matsuzaki O, Nagashima Y, Ozono S and Igarashi T; Japanese Society of Renal Cancer: Collecting duct (Bellini duct) renal cell carcinoma: A nationwide survey in Japan. J Urol 176: 40-43, 2006.
8. Antonelli A, Portesi E, Cozzoli A, Zanotelli T, Tardanico R, Balzarini P, Grigolato PG and Cosciani Cunico S: The collecting duct carcinoma of the kidney: A cytogenetical study. Eur Urol 43: 680-685, 2003.

9. Amin MB, Amin MB, Tamboli P, Javidan J, Stricker H, de-Peralta Venturina M, Deshpande A and Menon M: Prognostic impact of histologic subtyping of adult renal epithelial neoplasms: An experience of 405 cases. Am J Surg Pathol 26: 281-291, 2002.

10. Auguet T, Molina JC, Lorenzo A, Vila J, Sirvent JJ and Richart C: Synchronus renal cell carcinoma and Bellini duct carcinoma: A case report on a rare coincidence. World J Urol 18: 449-451, 2000.

11. Motzer RJ, Bander NH and Nanus DM: Renal-cell carcinoma. N Engl J Med 335: 865-875, 1996.

12. Hennessey DB, Thomas AZ and Lynch TH: Mixed collecting duct and renal cell carcinoma presenting with spinal cord compression. BMJ Case Rep 19: bcr2013008987, 2013.

13. Ockrim J, Tsiriopoulos I, Rees H, Mendoza N and Christmas TJ: Collecting duct carcinoma of the renal medulla presenting with paraplegia. Int Urol Nephrol 37: 465-466, 2005.

14. Schubert PT, Louw M, Scubert C, Eedes C and Theron J: A uncommon cause of haemoptysis: Metastatic collecting duct renal carcinoma. Diagn Cytopathol 39: 938-940, 2011.

15. Pickhardt PJ, Siegel CL and McLarney JK: Collecting duct carcinoma of the kidney: Are imaging findings suggestive of the diagnosis? AJR Am J Roentgenol 176: 627-633, 2001.

16. Wang L, Peng L, Hou T and Shi Y: Renal collecting duct carcinoma: Report of a case with unusual imaging findings regarding renal function. J Cancer Res Ther 9: 331-333, 2013.

17. Zhang J, Lefkowitz RA, Ishill NM, Wang L, Moskowitz CS, Russo P, Eisenberg H and Hricak H: Solid renal cortical tumors: Differentiation with CT. Radiology 244: 494-504, 2007. 